\title{
Fast Private Norm Estimation and Heavy Hitters
}

\author{
Joe Kilian $^{1, \star}$, André Madeira ${ }^{2}$, Martin J. Strauss ${ }^{3, \star \star}$, and Xuan Zheng ${ }^{4, \star \star \star}$ \\ ${ }^{1}$ Department of Computer Science, Rutgers University, Piscataway, NJ 08854 USA \\ jkilian@cs.rutgers.edu \\ ${ }^{2}$ Department of Computer Science, Rutgers University, Piscataway, NJ 08854 USA \\ amadeira@cs.rutgers.edu \\ ${ }^{3}$ Departments of Math and EECS, University of Michigan, Ann Arbor, \\ MI 48109 USA \\ martinjs@umich.edu \\ ${ }^{4}$ Department of EECS, University of Michigan, Ann Arbor, MI 48109 USA \\ xuanzh@eecs.umich.edu
}

\begin{abstract}
We consider the problems of computing the Euclidean norm of the difference of two vectors and, as an application, computing the large components (Heavy Hitters) in the difference. We provide protocols that are approximate but private in the semi-honest model and efficient in terms of time and communication in the vector length $N$. We provide the following, which can serve as building blocks to other protocols:

- Euclidean norm problem: we give a protocol with quasi-linear local computation and polylogarithmic communication in $N$ leaking only the true value of the norm. For processing massive datasets, the intended application, where $N$ is typically huge, our improvement over a recent result with quadratic runtime is significant.

- Heavy Hitters problem: suppose, for a prescribed $B$, we want the $B$ largest components in the difference vector. We give a protocol with quasi-linear local computation and polylogarithmic communication leaking only the set of true $B$ largest components and the Euclidean norm of the difference vector. We justify the leakage as (1) desirable, since it gives a measure of goodness of approximation; or (2) inevitable, since we show that there are contexts where linear communication is required for approximating the Heavy Hitters.
\end{abstract}

\section{Introduction}

Secure Multiparty Computation (SMC) has been studied for decades since 622]. Any protocol for computing a function can be converted, gate-by-gate, to a private protocol, in which no party learns anything from the protocol messages

\footnotetext{
* Supported in part by NSF grant CCF-0728937

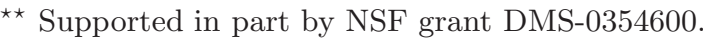

$\star \star \star$ Supported in part by NSF grant DMS-0354600. 
other than what can be inferred from the function's input/output relation. The computational overhead is at most polynomial in the size of the inputs.

In recent years, however, input sizes in many problems have grown to the point where "polynomial computational overhead" is too coarse a measure; both computation and communication should be minimized. For example, absent privacy concerns, applications may require that a protocol use at most polylogarithmic communication - this occurs in processing distributed internet traffic at line speeds or in performing data mining algorithms in very large datasets. General-purpose SMC may blow up communication exponentially, so additional techniques are needed. In one theoretical approach, individual protocols are designed for functions of interest such as database lookup 9187] and building decision trees [19]. Another important approach [21, converts any protocol into a private one with little communication blowup, but imposes a computational blowup that may be exponential in the communication complexity.

The approach we follow, which was introduced in [11, is to substitute an approximate function for the desired exact function. Many functions of interest have good approximations that can be computed efficiently both in terms of computation and communication. A caveat is that the traditional definition of privacy is no longer appropriate. Instead, a protocol $\pi$ computing an approximation $g$ to a function $f$ is a private approximation protocol [1] for $f$ if

- $\pi$ is a private protocol for $g$ in the traditional sense that the messages of $\pi$ leak nothing beyond what is implied by inputs and $g$; and,

- the output $g$ leaks nothing beyond what is implied by the inputs and $f$.

Several examples were given in [11]. Another important example was given in [15], where the authors provided an estimate $\|a-b\|_{\sim}$ (for integer-valued vectors $a$ and $b$ held by Alice and Bob respectively) as the first non-trivial example of polylogarithmic communication and polynomial computation. We will analyze and make use of this protocol for our results.

\subsection{Our Results}

Consider the general problem where Alice and Bob hold vectors, $a$ and $b$, of dimension $N$, and they want an efficient summary for the vector sum $c=a+b$. We analyze two problems in this setting.

First, we consider the Euclidean norm estimation problem, in which we wish to output a tight approximation to $\|c\|_{2}=\left(\sum_{i} c_{i}^{2}\right)^{1 / 2}$, the $l_{2}$ norm of vector $c$. The problem is a well-known building block for other protocols in the non-private setting, since it is used to estimate the skew of the data. A private protocol approximating $\|c\|_{2}$ using polylogarithmic communication in the vector length first appeared in [15]. Our results are based on their protocol; we strengthen its privacy guarantees and decrease its local computational costs. Specifically, we obtain a $O(N \log N)$ local computational cost versus the implied $\Omega\left(N^{2}\right)$, while keeping equivalent communication and round complexity costs.

Second, we consider the Euclidean approximate Heavy Hitters problem, in which there is a parameter, $B$, and the players ideally want $c_{\mathrm{opt}}$, the $B$ largest terms in $c$; i.e., the $B$ biggest values together with the corresponding indices. 
Unfortunately, finding $c_{\mathrm{opt}}$ exactly requires linear communication. Instead, the players use polylogarithmic communication (and polynomial work and $O(1)$ rounds) to output a vector $\widetilde{c}$ with $\|\widetilde{c}-c\|_{2} \leq(1+\epsilon)\left\|c_{\mathrm{opt}}-c\right\|_{2}$. In our protocol, the players learn nothing more than what can be deduced from $c_{\mathrm{opt}}$ and $\|c\|_{2}$. (We discuss below the significance of leaking $\|c\|_{2}$.) We can immediately use this result as a black box for taxicab approximate heavy hitters, i.e., finding $\widetilde{c}$ with $\|\widetilde{c}-c\|_{1} \leq(1+\epsilon)\left\|c_{\mathrm{opt}}-c\right\|_{1}$, leaking $c_{\mathrm{opt}}$ and $\|c\|_{2}$. We omit the development of this extension in the interest of space.

In the basic result, we give an at-most- $B$-term representation that is nearly as good (in the Euclidean sense) as the best $B$-term representation and leaks no more than the best $B$-term representation and the exact Euclidean norm. Although leaking the Euclidean norm represents a weaker result than not leaking it, actually (i) leaking $\|c\|_{2}$ is necessary and (ii) computing or approximating $\|c\|_{2}$ is desirable in some circumstances. First, we sketch a straightforward lower bound showing that, for some (reasonable) values of parameters $M, N, \ldots$, computing $\widetilde{c}$ leaking only $c_{\mathrm{opt}}$ requires $\Omega(N)$ communication. In fact, for some (artificial) classes of inputs, $\Omega(N)$ communication is needed unless $\|c\|_{2}$ itself is not only potentially leaked, but also actually computed exactly. On the other hand, one can regard the Euclidean norm as semantically interesting, so that we can regard the top $B$ terms together with the Euclidean norm as a compound, extended summary. In particular, since $\widetilde{c}$ is computed, leaking $\|c\|_{2}$ is equivalent to leaking $\|c\|_{2}^{2}-\|\widetilde{c}\|_{2}^{2}=\|\widetilde{c}-c\|_{2}^{2}$, i.e., the error in our representation, which is a useful and common desired result. Our protocol indeed can be modified to output an approximation $\|\widetilde{c}-c\|_{\sim}$ with $\|\widetilde{c}-c\|_{2} \leq\|\widetilde{c}-c\|_{\sim} \leq(1+\epsilon)\|\widetilde{c}-c\|_{2}$, so we can regard the protocol as solving two cascaded approximation problems: find a near-best representation $\widetilde{c}$, then find an approximation $\|\widetilde{c}-c\|_{\sim}$ to $\|\widetilde{c}-c\|_{2}$. It is natural to expect a protocol for $\widetilde{c}$ to leak $c_{\text {opt }}$ and a protocol for $\|\widetilde{c}-c\|_{\sim}$ to leak $\|\widetilde{c}-c\|_{2}$; while lower bounds prevent that, we can compute $\widetilde{c}$ and $\|\widetilde{c}-c\|_{\sim}$ simultaneously and guarantee that, overall, we leak only $c_{\mathrm{opt}}$ and $\|\widetilde{c}-c\|_{2}$.

\subsection{Related Work}

Other works in private communication-efficient protocols include the Private Information Retrieval problem [918]7, building decision trees [19], the set intersection and matching problem [12, and computing the $k^{\text {th }}$-ranked element 2 . The breakthrough work of [21] gives a general technique for converting any protocol into a private protocol with little communication overhead. However, this comes at the expense of local computational costs, which may increase exponentially. Thus, other general or application-specific techniques are needed.

The seminal work of [11] introduced the notion of private approximations and gave several protocols. Some negative results followed in 14 for approximations to NP-hard functions and more on NP-hard search problems appears in [5]. Recently, [15] gave a private approximation to the Euclidean norm that is central to our paper. Statistical work such as 8 , also addresses approximate summaries over large databases, but differs from our work in many parameters, such as the number of players and the allowable communication. 
Several papers address the Heavy Hitters problem, in a variety of contexts. Many of the needed ideas can be seen in [16] as well as in 341013. However, none are directly suitable when privacy is a concern.

\section{Road Map}

This paper is organized as follows. In Section 2, we present some necessary definitions used throughout the paper. We review private approximations in Section [3. In Section 4 we present our results for the private Euclidean norm estimation. Finally, in Section 5 we present our private approximate Euclidean Heavy Hitters protocol and some suitable lower bounds that motivate our results.

\section{Preliminaries}

Fix parameters $N, M, B, k$, and a distortion parameter $\epsilon$. In this paper, we consider only two players, Alice and Bob, holding input vectors $a$ and $b$ respectively, each of dimension $N$, and taking integer values in the range $[-M,+M]$. Let $k$ be a security and failure probability parameter and neg $(k, N)$ be an arbitrary negligible function of $k$ and $N$, i.e. a function that shrinks faster than any inverse polynomial in $k$ and $N$. We guarantee summaries whose error is at most the factor $(1+\epsilon)$ times the error of the best possible summary; and we will be interested in protocols that use communication poly $(B, \log (N), k, \log (M), 1 / \epsilon)$, local computation $\operatorname{poly}(B, N, k, \log (M), 1 / \epsilon)$, and $O(1)$ of rounds.

The Euclidean norm of a vector $c$ is $\|c\|_{2}=\left(\sum_{i} c_{i}^{2}\right)^{1 / 2}$. For the Heavy Hitters protocol, we are interested in summaries of size $B$ for the combined vector $c=$ $a+b$. For example, we are interested ideally in the largest $B$ terms of $c$. A vector $c$ is written $c=\left(c_{0}, c_{1}, c_{2}, \ldots, c_{N-1}\right)=\sum_{j} c_{j} \delta_{j}$, where $j$ is an index, $c_{j}$ is a value, $\delta_{j}$ is the vector that is 1 at index $j$ and 0 elsewhere, and $c_{j} \delta_{j}$, which can be implemented compactly and equivalently written as the pair $\left(j, c_{j}\right)$, is a term, in which $c_{j}$ is the coefficient. We compare terms by the magnitudes of their coefficients, breaking ties by the indices. That is, we will say that $\left(j, c_{j}\right)<\left(k, c_{k}\right)$ if $\left|c_{j}\right|<\left|c_{k}\right|$ or both $\left|c_{j}\right|=\left|c_{k}\right|$ and $j<k$. Thus all terms are strictly comparable. A heavy hitter summary is an expression of the form $\sum_{i \in \Lambda} \eta_{i} \delta_{i}$. If $|\Lambda|$ must be at most $B$, then the best heavy hitter summary $c_{\text {opt }}$ for a vector $c$ occurs where $\left\{\left(i, \eta_{i}\right): i \in \Lambda\right\}$ consists of the $B$ largest terms.

\subsection{Approximate Data Summaries}

A function $g$ is said to be an $\langle\epsilon, \delta\rangle$-approximation of $f$ if, for all inputs $x, \operatorname{Pr}[(1-$ $\epsilon) f(x) \leq g(x) \leq(1+\epsilon) f(x)] \geq 1-\delta$ holds for an approximation error $\epsilon \in(0,1)$ and confidence parameter $\delta \in(0,1)$. The probabilistic guarantee is over the randomness of $g$.

In the exact Heavy Hitters problem, we are given parameters $B$ and $N$ and the goal is to find the $B$ largest terms in a vector of dimension $N$. In the approximate Heavy Hitters problem, however, we want a summary $\widetilde{c}=\sum_{i \in \Lambda} \eta_{i} \delta_{i}$ such that $\|\widetilde{c}-c\| \leq(1+\epsilon)\left\|c_{\mathrm{opt}}-c\right\|$, where the norms are all Euclidean norms. 
In order to describe previous relevant algorithms, we first need some definitions. Fix a vector $c=\left(c_{0}, c_{1}, c_{2}, \ldots, c_{N-1}\right)=\sum_{0<i<N} c_{i} \delta_{i}$, whose terms are $t_{0}=\left(0, c_{0}\right), t_{1}=\left(1, c_{1}\right), \ldots, t_{N-1}=\left(N-1, c_{N-1}\right)$. Suppose the sequence $i_{0}^{\prime}, i_{1}^{\prime}, \ldots$ is a decreasing rearrangement of $c$, i.e., $t_{i_{0}^{\prime}}>t_{i_{1}^{\prime}}>\cdots>t_{i_{N-1}^{\prime}}$.

Definition 1 (Significant index). Let $I \subseteq[0, N)$ be a set of indices. Then $i$ is a $(I, \theta)$-significant index for $c$ if and only if $c_{i}^{2} \geq \theta \sum_{j \in I}\left|c_{j}\right|^{2}$.

That is, an index is significant if the corresponding value is large compared with all the other values. In some of the algorithms below, we will find the largest term (if it is sufficiently large), subtract it off, then recurse on the residual signal. This motivates the following definitions.

Definition 2 (Qualified index set). Fix parameters $\ell$ and $\theta$. The set $Q=$ $\left\{i_{0}^{\prime}, i_{1}^{\prime}, \ldots, i_{m-1}^{\prime}\right\}$ is a $(\ell, \theta)$-qualified index set for $c$ if and only if $(a) m \leq \ell$; (b) $\forall j \in[0, m-1], i_{j}^{\prime}$ is a $\left(\left\{i_{j}^{\prime}, i_{j+1}^{\prime}, \ldots, i_{N-1}^{\prime}\right\}, \theta\right)$-significant index; and $(c) i_{m}^{\prime}$ is NOT a $\left(\left\{i_{m}^{\prime}, i_{m+1}^{\prime}, \ldots, i_{N-1}^{\prime}\right\}, \theta\right)$-significant index.

That is, a qualified index set consists of the largest possible dimension $m$ for a prefix of $i_{0}^{\prime}, i_{1}^{\prime}, \ldots, i_{m-1}^{\prime}$ such that, for each $j<m$, we have $c_{i_{j}^{\prime}}^{2} \geq \theta\left(c_{i_{j}^{\prime}}^{2}+c_{i_{j+1}^{\prime}}^{2}+\right.$ $\left.c_{i_{j+2}^{\prime}}^{2}+\cdots+c_{i_{N-1}^{\prime}}^{2}\right)$. In particular, if the terms happen to be in decreasing order to begin with, i.e., if $\left|c_{0}\right|>\left|c_{1}\right|>\cdots$, then a qualified index set is $\{0,1,2, \ldots, m-1\}$ for the largest $m$ such that, for each $j<m$, we have $c_{j}^{2} \geq \theta\left(c_{j}^{2}+c_{j+1}^{2}+c_{j+2}^{2}+\right.$ $\left.\cdots c_{N-1}^{2}\right)$. Note that for each $\ell, \theta$, and vector $c$, there is only one $(\ell, \theta)$-qualified index set for $c$. We use $Q_{c, \ell, \theta}$ to denote it and sometimes write $Q_{\ell, \theta}$ when $c$ is understood. The following Proposition is then straightforward.

Proposition 3. For any $\theta_{1}<\theta_{2}, Q_{\ell, \theta_{2}}$ set is a subset of $Q_{\ell, \theta_{1}}$.

Proposition 4. Fix parameters $N, M, B, k, \epsilon$ and the vector $c$ as above. If $\widetilde{c}=$ $\sum_{i \in Q_{c, B, \frac{\epsilon}{B(1+\epsilon)}}} c_{i} \delta_{i}$, then $\|\tilde{c}-c\|_{2}^{2} \leq(1+\epsilon)\left\|c_{\mathrm{opt}}-c\right\|_{2}^{2}$.

Proof. Assume without loss of generality that $\left|c_{0}\right|>\left|c_{1}\right|>\cdots$ and let $q=$ $\left|Q_{c, B, \frac{\epsilon}{B(1+\epsilon)}}\right|$. If $q=B$, then $\widetilde{c}=c_{\text {opt }}$ and we are done. Otherwise we have $\|\widetilde{c}-c\|_{2}^{2}$ $=\sum_{q \leq i<B}\left|c_{i}\right|^{2}+\left\|c_{\mathrm{opt}}-c\right\|_{2}^{2} \leq B\left|c_{q}\right|^{2}+\left\|c_{\mathrm{opt}}-c\right\|_{2}^{2} \leq \frac{\epsilon}{1+\epsilon}\|\widetilde{c}-c\|_{2}^{2}+\left\|c_{\mathrm{opt}}-c\right\|_{2}^{2}$, whence $(1-\epsilon /(1+\epsilon))\|\widetilde{c}-c\|_{2}^{2} \leq\left\|c_{\mathrm{opt}}-c\right\|_{2}^{2}$. The result follows.

The algorithms below work from a linear sketch of a vector.

Definition 5 (Sketch of a vector). Given a vector $c$, a linear sketch of $c$ is $R c$, where $R$ is a random matrix, called the measurement matrix, generated from a prescribed distribution

In our case, as is typical, the matrix $R$ is a pseudorandom matrix that can be generated from a short pseudorandom seed. We use sketching for the NORM_ESTIMATION protocol (Protocol 1 in Section 4), in which the generator needs to be secure against small space, and a different measurement matrix in 
the non-private Euclidean Heavy Hitters protocol, where, e.g., pairwise independence suffices for the pseudorandom number generator.

An algorithm in connection with the approximate Euclidean Heavy Hitters problem satisfying the following is known [13]:

Theorem 6. Fix $N, M, B, k, \epsilon$ as above, and $\theta \geq \operatorname{poly}(\log (N), \log (M)$, $B, k, 1 / \epsilon)^{-1}$. There is a distribution on sketch matrices $R$ and a corresponding algorithm that, from $R$ and sketch $R c$ of a vector $c$, outputs a superset of $Q_{c, B, \theta}$, in time poly $(\log (N), \log (M), B, k, 1 / \epsilon)$.

In particular, the number or rows in $R$ and the size of the output is bounded by the expression poly $(\log (N), \log (M), B, k, 1 / \epsilon)$ in accordance with the time bound on the algorithm. The algorithm admits efficient Secure Function Evaluation protocols, and can be modified to run privately in poly $(\log (N), \log (M), B, k, 1 / \epsilon)$ time. Note that the algorithm returns a superset of $Q_{c, B, \theta}$ but that even $Q_{c, B, \theta}$ itself suffices for a good approximation.

\subsection{Private Two-Player Protocol}

SMC allows two or more parties to evaluate a previously-agreed-upon function of their inputs, while hiding their inputs from each other. Here, we assume that all parties are computationally bounded and semi-honest, meaning they follow the protocol but may keep message histories in an attempt to learn more than is prescribed. The adversary is thus passive and can't modify the behavior of corrupted parties. In [21], the authors have shown how to transform a semihonest protocol into a protocol secure in the malicious model, where parties deviate from the protocol arbitrarily using a different input or outputing the wrong answer, or even exiting from the protocol prematurely. Therefore, we assume parties are semi-honest for the remainder of the paper.

Formally, a two-party computation is specified by a (possibly randomized) mapping $g$ from a pair of inputs $(a, b) \in\{0,1\}^{*} \times\{0,1\}^{*}$ to a pair of outputs $(c, d) \in\{0,1\}^{*} \times\{0,1\}^{*}$. Let $\pi=\left(\pi_{A}, \pi_{B}\right)$ be a two-party protocol computing $g$. Consider the probability space induced by the execution of $\pi$ on input $\mathbf{x}=(a, b)$ (induced by the independent choices of random inputs $\left.r_{A}, r_{B}\right)$. Let view $_{A}^{\pi}(\mathbf{x})$ (resp., view ${ }_{B}^{\pi}(\mathbf{x})$ ) denote the entire view of Alice (resp., Bob) in this execution, including her input, random input, and all messages she has received. Let output $\pi_{A}^{\pi}(\mathbf{x})$ (resp., output ${ }_{B}^{\pi}(\mathbf{x})$ ) denote Alice's (resp., Bob's) output. Note that the above four random variables are defined over the same probability space. Two distributions (or ensembles) $\mathcal{D}_{1}$ and $\mathcal{D}_{2}$ are said to be computationally indistinguishable with security parameter $k, \mathcal{D}_{1} \stackrel{c}{\equiv} \mathcal{D}_{2}$, if, for any $X_{1} \sim \mathcal{D}_{1}$ and $X_{2} \sim \mathcal{D}_{2}$ and, for any family of polynomial-size circuits $\left\{C_{k}\right\}$, we have $\left|\operatorname{Pr}\left(C_{k}\left(X_{1}\right)=1\right)-\operatorname{Pr}\left(C_{k}\left(X_{2}\right)=1\right)\right| \leq \operatorname{neg}(k)$.

Definition 7 (Private two-party protocol). Let $X$ be the set of all valid inputs $\mathbf{x}=(a, b)$. A protocol $\pi$ is a private protocol computing $g$ if the following properties hold: 
Correctness. The joint outputs are distributed according to $g(a, b)$. Formally,

$$
\left\{\left(\text { output }_{A}^{\pi}(\mathbf{x}), \text { output }_{B}^{\pi}(\mathbf{x})\right)\right\}_{\mathbf{x} \in X} \equiv\left\{\left(g_{A}(\mathbf{x}), g_{B}(\mathbf{x})\right)\right\}_{\mathbf{x} \in X},
$$

where $\left(g_{A}(\mathbf{x}), g_{B}(\mathbf{x})\right)$ is the joint distribution of the outputs of $g(\mathbf{x})$.

Privacy. There exist probabilistic polynomial-time algorithms $\mathcal{S}_{A}, \mathcal{S}_{B}$, also known as simulators, such that:

$$
\begin{array}{r}
\left\{\left(\mathcal{S}_{A}\left(a, g_{A}(\mathbf{x})\right), g_{B}(\mathbf{x})\right)\right\}_{\mathbf{x}=(a, b) \in X} \stackrel{c}{\equiv}\left\{\left(\operatorname{view}_{A}^{\pi}(\mathbf{x}), \operatorname{output}_{B}^{\pi}(\mathbf{x})\right)\right\}_{\mathbf{x} \in X} \\
\left\{\left(g_{A}(\mathbf{x}), \mathcal{S}_{B}\left(b, g_{B}(\mathbf{x})\right)\right\}_{\mathbf{x}=(a, b) \in X} \stackrel{c}{\equiv}\left\{\operatorname{output}_{A}^{\pi}(\mathbf{x}), \operatorname{view}_{B}^{\pi}(\mathbf{x})\right)\right\}_{\mathbf{x} \in X}
\end{array}
$$

Yao, in its seminal work 22], provided a general technique:

Proposition 8 (General-Purpose SMC [22]). Two parties holding inputs $x$ and $y$ can privately compute any circuit $C$ with communication and computation $O(k(|C|+|x|+|y|))$, where $k$ is a security parameter, in $O(1)$ rounds.

We also require the following notion of evaluating a circuit with ROM securely. In this context, the $i^{\text {th }}$ party has a table $R_{i} \in\left(\{0,1\}^{r}\right)^{s}$, a function of its inputs. Then, the circuit has lookup gates, which on inputs $(i, j)$ returns $R_{i}[j]$.

Proposition 9 (Secure Circuit with ROM [21]). If $C$ is a circuit with ROM, then it can be securely evaluated with $O(k|C| T(r, s))$ communication in $O(1)$ rounds, where $T(r, s)$ is the communication of 1-out-of-s Oblivious Transfer (OT) protocol on words of size $r$.

We will need the following standard definitions for our results in Section 5 .

Definition 10 (Additive Secret Sharing). An intermediate value $x$ of a joint computation is said to be secret shared between Alice and Bob if Alice holds $r$ and Bob holds $x-r$, modulo some large prime, where $r$ is a random number independent of all inputs and outputs.

Definition 11 (Private Sample Sum). At the start, Alice holds a vector a of dimension $N$ and Bob holds a vector $b$. Alice and Bob also hold a secret sharing of an index $i$. At the end, Alice and Bob hold a secret sharing of $a_{i}+b_{i}$.

That is, neither the index $i$ nor the value $a_{i}+b_{i}$ becomes known to the parties. Efficient protocols for this problem can be found (or can be constructed immediately from related results) in 2111, under various assumptions about the existence of Private Information Retrieval, such as in [7.

Proposition 12. There is a protocol PRIVATE-SAMPLE-SUM for the Private Sample Sum problem that requires $\operatorname{poly}(N, k)$ computation, $\operatorname{poly}(\log (N), k)$ communication, and $O(1)$ rounds.

\section{Private Approximations}

In this Section, we review the notion of private approximations introduced in [1]. 
Definition 13 (Private Approximation Protocol (strict sense) [11]). A two-party private approximation protocol for a deterministic, common-output function $g$ on inputs $a$ and $b$ is strict if it computes an approximation $\widetilde{g}$ to $g$ such that: (a) $\widetilde{g}$ is a good approximation to $g$ (in the appropriate sense); (b) $\pi$ is a private protocol for $\widetilde{g}$ in the traditional sense (Definition 7 ); and (c) (Functional Privacy) there exists a probabilistic polynomial-time (PPT) simulator $\mathcal{S}$ s.t.:

$$
\{\mathcal{S}(g(\mathbf{x}))\}_{\mathbf{x}=(a, b) \in X} \stackrel{c}{\equiv} \widetilde{g}(\mathbf{x}) .
$$

In the case the output to both parties is a deterministic function, a (weakly) equivalent definition is as follows, known as the "liberal" definition in [11]:

Definition 14 (Private Approximation Protocol (liberal sense) [11]). A two-party private approximation protocol for a deterministic, common-output function $g$ on inputs $a$ and $b$ is liberal if it computes an approximation $\widehat{g}$ to $g$ such that: (a) $\widehat{g}$ is a good approximation to $g$ (in the appropriate sense); (b) $\pi$ is a private protocol for $\widehat{g}$, with correctness as in Definition 7 and privacy as in existing PPT simulators $\mathcal{S}_{A}$ and $\mathcal{S}_{B}$ such that:

$$
\begin{aligned}
& \left\{\mathcal{S}_{A}(a, g(\mathbf{x}))\right\}_{\mathbf{x}=(a, b) \in X} \stackrel{c}{\equiv}\left\{\operatorname{view}_{A}^{\pi}(\mathbf{x})\right\}_{\mathbf{x} \in X} \\
& \left\{\mathcal{S}_{B}(b, g(\mathbf{x}))\right\}_{\mathbf{x}=(a, b) \in X} \stackrel{c}{\equiv}\left\{\operatorname{view}_{B}^{\pi}(\mathbf{x})\right\}_{\mathbf{x} \in X} ;
\end{aligned}
$$

and (c) (Functional Privacy) there exists a PPT simulator $\mathcal{S}$ such that:

$$
\{\mathcal{S}(g(\mathbf{x}))\}_{\mathbf{x}=(a, b) \in X} \stackrel{c}{\equiv} \widehat{g}(\mathbf{x}) .
$$

We elaborate on a general technique, originally sketched in [1], to construct a private protocol in the strict sense given a private protocol in the liberal sense. We also show the intuitive fact that the converse always holds.

Proposition 15 (equivalency between liberal and strict definitions). Any private approximation protocol in the liberal sense requiring only polylogarithmic communication complexity can be transformed into a private approximation protocol in the strict sense with the same asymptotic communication complexity, local computational costs, and rounds. The converse holds true as well.

Proof. Let $\widehat{g}$ and $\widetilde{g}$ be $\langle\epsilon, \delta\rangle$-approximations of $g$; and $\widehat{\pi}$ and $\widetilde{\pi}$ be private protocols computing $\widehat{g}$ and $\widetilde{g}$ in the liberal and strict sense respectively. Now, suppose there are simulators in the strict sense. Then, putting $\widehat{g}=\widetilde{g}$, a simulator for the liberal definition can be constructed by simulating $\widehat{g}(a, b)=\widetilde{g}(a, b)$ from $g(a, b)$ using the hypothesized simulator for functional privacy, then simulating Alice's (or Bob's) view from $\widehat{g}(a, b)$ and $a$ (or $b$ ) using the hypothesized strict simulator.

In the other direction, suppose there is a simulator in the liberal definition. Let $\tau$ be a transcript of Alice's view except for input $a$. Define $\widetilde{g}=\widehat{g} \cdot \tau$ to be $\widehat{g}$ with $\tau$ encoded into its low-order bits. We assume that this kind of encoding into approximations can be accomplished without significantly affecting the goodness of approximation; in fact, we will assume that the value represented does not change at all, even if the "approximate" value is zero - that is, $\tau$ is auxiliary data 
rather than an actual part of the value of $\widetilde{g}$. Furthermore, since $\tau$ is polylogarithmically bounded in the input size, the communication overhead of $\widetilde{g}$ over $\widehat{g}$ is at most the size of $\tau$, since a protocol for $\widehat{g}$ also serves as a protocol for $\widetilde{g}$. It is trivial to simulate the protocol messages given $a$ and $\widetilde{g}$. Use the hypothesized simulator in the liberal definition to show functional privacy of $\widetilde{g}$.

In Section 4, we apply the technique above of encoding the transcript into the low-order bits to the NORM_ESTIMATION protocol from [15], originally presented in the liberal definition, to achieve a more secure version abiding by the strict definition. Furthermore, our Heavy Hitters result in Section 5 is formally proven in the strict sense using the same idea.

\section{Private Euclidean Norm Estimation}

We consider the setting in which Alice and Bob hold integer-valued vectors $a$ and $b$ respectively, each of dimension $N$. In [15, the authors provided a protocol for privately approximating the Euclidean norm of the vector difference $\|c\|=$ $\|a-b\|$ as well as the similar vector sum. Before we present our enhancements, it is instructive to review the inner workings of their protocol and its guarantees, given in Protocol[1 and Proposition 16 respectively.

\section{NORM_ESTIMATION}

Inputs: $N$-dimensional vectors $a$ and $b$ with integer values in the range $[-M, M]$.

Output: An $\langle\epsilon, \delta\rangle$-approximation of $\|c\|^{2}$, where $c=a-b$.

1. Alice and Bob exchange a seed of a pseudorandom generator $G$ and generate a pseudorandom orthonormal matrix $A$.

2. Set $T=T_{\max }=N M^{2}$

3. Repeat (\{Assertion: $\left.\left.\|c\|^{2} \leq T\right\}\right)$

(a) $\forall j \in[l]$, a secure circuit with ROM (with lookup tables on $A a$ and $A b$ ) independently generates random coordinates $i_{j}$, computes $(A x)_{i_{j}}^{2}$, and independently generates $z_{j}$ from a $\operatorname{Bernoulli}\left(N(A x)_{i_{j}}^{2} /(T B)\right)$ distribution.

(b) $T=T / 2$

4. Until $\sum_{i} z_{i} \geq l /(4 B)$ or $T<1$

5. Output $E=(2 T B) / l \cdot \sum_{i} z_{i}$ as an estimate of $\|c\|^{2}$.

Protocol 1. Private approximation protocol of the square $l_{2}$ difference 15

Proposition 16. (Private $l_{2}$ approximation [15]) Suppose Alice and Bob have integer-valued vectors $a$ and $b$ in $[-M, M]^{N}$ and let $c=a-b$. Fix distortion $\epsilon$ and security parameter $k$. There is a protocol NORM_ESTIMATION that computes an approximation $\|c\|_{\sim}$ to the Euclidean norm of the vector difference, $\|c\|_{2}$, such that it (a) outputs $\frac{1}{1+\epsilon}\|c\|_{2} \leq\|c\|_{\sim} \leq\|c\|_{2}$; (b) requires $\operatorname{poly}(k \log (M) N / \epsilon)$ local computation, $\operatorname{poly}(k \log (M) \log (N) / \epsilon)$ communication, and $O(1)$ rounds; and (c) is a private approximation protocol for $\|c\|_{2}$ in the liberal sense.

Furthermore, the protocol's only access to $a$ and $b$ is through the matrix-vector products $A a$ and $A b$, where $A$ is a pseudorandom matrix known to both players. 
The access is possible through evaluating a circuit with ROM securely; i.e. a circuit with lookup gates on inputs $A a$ and $A b$ (see Proposition 9).

Observe that although the communication complexity of this protocol is low, the computational complexity of their protocol is quadratic in the vector dimension $N$. The protocol multiplies the matrix $A$, which has $\Theta\left(N^{2}\right)$ degrees of freedom by the input vectors $a$ and $b$, thus requiring $\Omega\left(N^{2}\right)$ computations. Before we present our enhancements to Protocol 1, we first sketch the intuition behind its construction, correctness and privacy guarantees.

In [20], the authors have shown that picking a random $N \times N$ orthonormal matrix $A$ from a distribution defined by the Haar measure ensures that each component of $A x$, for any vector $x$, is tightly concentrated around its root mean square, $\|x\| / \sqrt{N}$. Formally, there exists a $c>0$ such that

$$
\operatorname{Pr}\left[\left|(A x)_{i}\right| \geq t\|x\| / \sqrt{N}\right] \leq e^{-c t^{2}}
$$

holds for any $i=1, \ldots, N$, any $t>1$ and any $x \in \mathbb{R}^{N}$. This transformation ensures that the "mass" of vector $x$ is uniformly spread among the $N$ coordinates while preserving the vector norm, i.e. $\|x\|=\|A x\|$. Protocol 1 uses this fact and constructs $A$ using pseudorandom generators instead, guaranteeing nonetheless that $\left(1-2^{-\Theta(k)}\right)\|x\|^{2} \leq\|A x\|^{2} \leq\|x\|^{2}$ holds except with $\operatorname{neg}(k, N)$ probability. Note that with each component tightly concentrated around the root mean square, one can construct an unbiased sample estimator which is an $\langle\epsilon, \delta\rangle$ approximation by straightforward application of Chernoff bounds. However, to achieve privacy, the protocol must sample the coordinates $(A x)_{i}$ obliviously as to prevent either party from learning the sampled values (it does so by using a secure circuit with ROM; see Section 2.2). Furthermore, the protocol ensures that the final estimate $E$ depends only on $\|x\|$ by using Bernoulli trials to squash the higher moments of $E$, thus preventing non-simulatable information from leaking. In particular, this also achieves Functional Privacy as needed in Definition 14. For its correctness argument, the protocol guarantees that each $z_{j}$ has enough information to approximate $l_{2}(x)$ tightly by scaling the Bernoulli trials by a loop variable $T$ and exiting the loop when the sum of the trials is large enough for tight estimation. We refer the reader to [15] for complete analysis of Protocol[1]

\subsection{Faster Approximation}

As argued in the last Section, the computation bottleneck of Protocol 1 is the multiplication of the pseudorandom matrix $A$ by the input vectors $a$ and $b$. Computing $A a$ (and $A b$ ) requires $\Omega\left(N^{2}\right)$ due to the $\Theta\left(N^{2}\right)$ degrees of freedom of matrix $A$. We recall that this multiplication step is crucial for both the correctness and privacy guarantees. The matrix transformation ensures that the "mass" of the vector is uniformly spread among all coordinates while preserving the norm. Such process allows a circuit to sample logarithmic many coordinates for a tight estimation of the same norm. Preserving the norm ensures that the Bernouilli trials can be simulated for the privacy proof. 
We perform a similar but faster matrix transformation on Alice and Bob's input vectors. The transformation also spreads the "mass" of the vector uniformily and preserves the vector norms as required by the correctness and privacy arguments of Protocol1 However, our matrix multiplication takes only $O(N \log N)$ time as opposed to $\Omega\left(N^{2}\right)$.

Our approach, based on the technique of Ailon and Chazelle [1, is to randomly choose from a "sufficiently random" family of easily computable orthonormal transformations, as follows. Given a vector $x=\left(x_{1}, x_{2}, \ldots, x_{N}\right)$, we flip the sign of each $x_{i}$ independently with probability $1 / 2$ and then apply a Hadamard transform to it, yielding a new vector $x^{*}$. Thus we choose uniformly from a family of $2^{N}$ linear transformations, each corresponding to a choice of which variables to sign-flip. Since the Hadamard transform is orthonormal and can be computed in $O(N \log N)$ time, it follows that each transformation in our family is orthonormal and computable in $O(N \log N)$ time, as flipping the sign of a variable is an orthonormal transformation with trivial computational overhead.

Next, we observe that each $x_{j}^{*}$, viewed in isolation, is a random linear combination of signed and unsigned $x_{i}$ 's, scaled by $1 / \sqrt{N}$. We prove that each $x_{i}^{*}$ is not larger than the root mean square of $x$, or $\|x\| / \sqrt{N}$, with high probability. Thus, we achieve a similar bound for each coordinate $(A x)_{i}$ as in equation Eq. (1), which suffices for the correctness and privacy proofs of the original protocol.

The following lemma summarizes the above discussion and claims.

Lemma 1. Let $x$ and $x^{\prime}$ be vectors of dimension $N$, with each $x_{i}^{\prime}$ being the result of flipping the signal of the corresponding $x_{i}$ with probability 1/2. Then, for any $\lambda>0$, applying a Hadamard transform to vector $x^{\prime}$, yielding $x^{*}=\frac{1}{\sqrt{N}} H_{N} x^{\prime}$, where $H_{N}$ is the $N \times N$ Hadamard matrix, we have that

$$
\operatorname{Pr}\left[\left|x_{i}^{*}\right| \geq \lambda \frac{|x|}{\sqrt{N}}\right] \leq 2 e^{-\lambda^{2} / 2} .
$$

Proof. We analyze the case for a particular $x_{j}^{*}$, for $j \in[1, N]$. Let $Z_{1}, \ldots, Z_{N}$ be independent variables such that $Z_{i}=\left(\zeta_{i} x_{i}\right) / \sqrt{N}$, where $\zeta_{i} \in_{R}\{+1,-1\}$. Here, $\epsilon_{R}$ denotes drawing each $\zeta_{i}$ independently and uniformily at random. Note that $E\left[Z_{i}\right]=0$. Now, let $S=\sum_{i}^{N} Z_{i}$. We then define a martingale sequence $X_{0}, X_{1}, \ldots, X_{N}$ by setting $X_{0}=E[S]$ and, for $i \in[1, N], X_{i}=$ $E\left[S \mid Z_{1}, \ldots, Z_{i}\right]$. We now apply Azuma's inequality as follows. Recall that for a martingale sequence $X_{0}, X_{1}, \ldots, X_{N}$ s.t. $\left|X_{k}-X_{k-1}\right| \leq c_{k}, \operatorname{Pr}\left[\left|X_{t}-X_{0}\right| \geq \lambda\right] \leq$ $2 \exp \left(-\frac{\lambda^{2}}{2 \sum_{k=1}^{t} c_{k}^{2}}\right)$ for any $t \geq 0$ and any $\lambda>0$. For our martingale difference sequence let $c_{k}=\left|X_{k}-X_{k-1}\right|$ and thus we get

$$
\operatorname{Pr}\left[\left|X_{N}-X_{0}\right| \geq \lambda \frac{|x|}{\sqrt{N}}\right] \leq 2 \exp \left(-\frac{\lambda^{2}}{2} \frac{|x|^{2}}{N} \frac{1}{\sum_{k=1}^{N} c_{k}^{2}}\right) .
$$

Thus, to prove Eq. (2) it suffices to show that $\sum_{k=1}^{N} c_{k}^{2} \leq|x|^{2} / N$. Note that $X_{k}-X_{k-1}=Z_{k}=\left(\zeta_{k} x_{k}\right) / \sqrt{N}$, and thus $\sum_{k=1}^{N} c_{k}^{2}=\sum_{k=1}^{N}\left(X_{k}-X_{k-1}\right)^{2}=$ $\sum_{k=1}^{N}\left(\frac{\zeta_{k} x_{k}}{\sqrt{N}}\right)^{2}=\frac{|x|^{2}}{N}$. Therefore, applying it to Eq. (3) guarantees Eq. (2). 


\subsection{More Secure Approximation}

In 15, the authors have shown that Protocol 1 is secure in the liberal sense. They provided the NORM_ESTIMATION simulator that guarantees both functional privacy and private computation of protocol $\widehat{\pi}$ (Protocol 1) computing an approximation $\widehat{g}$ of $g=\|x\|^{2}$. Their simulator receives the exact output $\|x\|^{2}$ for generating the protocol transcripts. To be secure in the strict sense, besides showing functional privacy, one must provide a simulator that is able to produce computationally indistingishable views from Alice's and Bob's without access to the exact output, but only to the approximation output $\widehat{g}$ (see Definition [13). The original NORM_ESTIMATION simulator from 15 is shown next.

\section{NORM_ESTIMATION simulator}

Input: $\|x\|^{2}$

Output: a computationally indistinguishable distribution from Protocol $1 \square$

1. Generate a random seed of $G$

2. Set $T=T_{\max }=n M^{2}$

3. Repeat:

(a) $\forall j \in[l]$, independently generate $z_{j}$ from a Bernoulli $\left(\|x\|^{2} /(T B)\right)$ distribution

(b) $T=T / 2$

4. Until $\sum_{i} z_{i} \geq l /(4 B)$ or $T<1$

5. Output $E=(2 T B) / l \cdot \sum_{i} z_{i}$

Simulator 1. The NORM_ESTIMATION simulator from 15

Simulator 1 above guarantees that the probabilities of the Bernoulli trials from the real and simulated views differ only by neg $(k, N)$. Thus, given access to the exact and approximate outputs, $g(x)$ and $\widehat{g}(x)$ respectively, all messages exchanged - the seed, the oblivious transfer (OT) invocations by the secure circuit, and the output - are simulatable. Specifically, the final value of $T$ is also simulatable. Note that simulating the final value of $T$ is crucial to the privacy argument. If the number of invocations made by the secure circuit differ between the real and simulated views, the distribution on the resulting transcripts will no longer be indistinguishable. Furthermore, observe that the exact output $g(x)$ is necessary for simulating such number of steps since its magnitude dictates the loop exit condition. Clearly, using $\widehat{g}(x)=(1 \pm \epsilon)\|x\|^{2}$ to replace $g(x)=\|x\|^{2}$ in the Bernoulli trials would make the probabilities differ by a factor in the order of $O(\epsilon)$, a non-negligible factor in our security setting; i.e. we expect $O\left(2^{-\Theta(k)}\right)$.

Nonetheless, we show how can we transform this liberal protocol into a strict one, by using the general technique outlined in Section 3. We define a new approximation function $\widetilde{g}$ based on $\widehat{g}$. Let $\widehat{\tau}$ denote the transcript of protocol $\widehat{\pi}$ and let $\widetilde{g}=\widehat{g} \cdot \widehat{\tau}$, meaning that the output of the new approximation function is the output of the original approximation function $\widehat{g}$ concatenated to the entire transcript of protocol $\widehat{\pi}$ (one can view $\widehat{\tau}$ as encoded into the low-order bits of the approximation - in which case we assume the goodness of approximation is 
not substantially changed - or, alternatively, as auxiliary data and not part of the output itself). The transcript $\widehat{\tau}$ in this case is just a concatenation of the seed used for the pseudorandom generator, all OT invocations by the secure circuit, and the final approximate output. Thus, the communication costs at most doubled; and thus still remain asymptotically poly $(k \log (M) \log (N) / \epsilon)$.

Let the new protocol $\widetilde{\pi}$ computing $\widetilde{g}$ be identical to $\widehat{\pi}$ with the additional output of $\widehat{\tau}$ along with $\widehat{g}$. It remains to show that $\widetilde{\pi}$ can be privately computed. We thus create Simulator 2, which clearly generates indistinguishable views for Alice and Bob, since all messages exchanged in protocol $\tilde{\pi}$ are simulated properly: the random seed messages, a matching number of OT calls as well as the final output. Finally, it is clear that $\widetilde{g}$ is functionally private to $g$ since one can use the NORM_ESTIMATION simulator to output $\widehat{\tau}$ along with $\widehat{g}(x)$ given only $g(x)=\|x\|$.

\section{$\tilde{\pi}$ simulator}

Input: $\quad \widetilde{g}(x)=\widehat{g}(x) \cdot \widehat{\tau}$

Output: a computationally indistinguishable transcript from $\tilde{\pi}(x)$

1. Extract $\widehat{g}(x)$ and $\widehat{\tau}$ from the input $\widetilde{g}(x)=\widehat{g}(x) . \widehat{\tau}$

2. Extract the random seed for the pseudorandom generator from $\widehat{\tau}$ and send it to the other party.

3. Simulate the OT calls from Step 3 in Protocol 1 $\square$ y playing back the messages exchanged in $\widehat{\tau}$.

4. Output $\widetilde{g}(x)$

\section{Simulator 2. Simulator for $\tilde{\pi}$}

\section{Private Euclidean Heavy Hitters}

Consider the same input setting from the previous Section. Here, both parties want to learn a representation $\widetilde{c}=\sum_{t \in T_{\text {out }}} t$ such that $\|c-\widetilde{c}\|_{2}^{2} \leq(1+$ $\epsilon)\left\|c-c_{\mathrm{opt}}\right\|_{2}^{2}$ and such that at most $c_{\mathrm{opt}}$ and $\|c\|_{2}$ is revealed. Unless otherwise stated, we consider the private Euclidean Heavy Hitters problem as simply the private Heavy Hitters problem. A protocol is given in Figure 2 .

\subsection{Analysis}

First, to gain intuition, we consider some easy special cases of the protocol's operation. For our analysis, assume that the terms in $c$ are already positive and in decreasing order, $c_{0}>c_{1}>\cdots>c_{N-1}>0$. We will be able to find the coefficient value of any desired term, so we focus on the set of indices. Let $I_{\text {opt }}=\{0,1,2, \ldots, B-1\}$ denote the set of indices for the optimal $B$ terms. The set $I$ of indices is defined in Figure 2. Thus $Q_{c, B, \theta} \subseteq Q_{c, B, \frac{\theta}{1+\epsilon}} \subseteq I_{\text {opt }}$ and $Q_{c, B, \frac{\theta}{1+\epsilon}} \subseteq I$.

The ideal output is $I_{\mathrm{opt}}$, though any superset of $Q_{c, B, \theta}$ suffices to get an approximation with error at most $(1+\epsilon)$ times optimal. This includes the set 
$I \supseteq Q_{c, B, \theta}$ that the non-private algorithm has recovered. The set $I_{B}$ of the largest $B$ terms indexed by $I$ contains $Q_{c, B, \theta}$, so $I_{B}$ is a set of at most $B$ terms with error at most $(1+\epsilon)$ times optimal. If $\left|Q_{c, B, \theta}\right|=B$, then $I_{B}=Q_{c, B, \theta}=I_{\text {opt }}$, and $I_{B}$ is a private and correct output.

PRIVATE_HEAVY_HITTERS

- Known parameters: $N, M, B, \epsilon, k$, which determine $\theta=\frac{\epsilon}{B(1+\epsilon)}$ and $B^{\prime}$.

- Inputs: $N$-dimensional vectors $a$ and $b$ with integer values in the range $[-M, M]$.

- Output: With probability at least $1-2^{-k}$, a set $T_{\text {out }}$ of at most $B$ terms, such that $\left\|c-\sum_{t \in T_{\text {out }}} t\right\|_{2}^{2} \leq(1+\epsilon)\left\|c-\sum_{t \in T_{\text {opt }}} t\right\|_{2}^{2}$.

1. Exchange pseudorandom seeds (in the clear). Generate measurement matrices $R_{1}$ and $R_{2}$. Alice locally constructs sketches $R_{1} a$ and $R_{2} a=\left(R_{2}^{0} a, R_{2}^{1} a, \ldots R_{2}^{B-1} a\right)$, where the matrix $R_{1}$ is used for a non-private Euclidean Heavy Hitters and the matrix $R_{2}=\left(R_{2}^{0}, R_{2}^{1}, \ldots, R_{2}^{B-1}\right)$ is used for $B$ independent repetitions of NORM_ESTIMATION. Bob similarly constructs $R_{1} b$ and $R_{2} b$.

2. Using general-purpose SMC, do

- Use an existing (non-private) Euclidean Heavy Hitters protocol to get, from $R_{1} a$ and $R_{1} b$, a secret-sharing of a superset $I$ of $Q_{c, B, \frac{\theta}{1+\epsilon}}$, in which $I$ has exactly $B^{\prime} \leq \operatorname{poly}(\log (N), \log (M), B, k, 1 / \epsilon)$ indices. (Pad, if necessary.)

3. Use PRIVATE-SAMPLE-SUM to compute, from $I, a$, and $b$, secret-shared values for each index in $I$. Let $T$ denote the corresponding set of secret-shared terms. (Both the index and value of each term in $T$ is secret shared.) Enumerate $I$ as $I=$ $\left\{i_{0}, i_{1}, \ldots\right\}$ with $t_{i_{0}}>t_{i_{1}}>\cdots$.

4. Using SMC, do

- for $j=0$ to $B-1$

(a) From $R_{2}^{j}, R_{2}^{j} a, R_{2}^{j} b, t_{0}, t_{1}, \ldots, t_{i_{j-1}}$, sketch $r_{j}=c-\left(t_{i_{0}}+t_{i_{1}}+\cdots+t_{i_{j-1}}\right)$ as $R_{2}^{j} r_{j}=\left(R_{2}^{j} a+R_{2}^{j} b-R_{2}^{j}\left(t_{i_{0}}+t_{i_{1}}+\cdots+t_{i_{j-1}}\right)\right)$.

(b) use NORM_ESTIMATION to estimate $\left\|r_{j}\right\|_{2}^{2}$ as $\left\|r_{j}\right\|_{\sim}^{2}$, satisfying $\frac{1}{1+\epsilon}\left\|r_{j}\right\|_{2}^{2} \leq$ $\left\|r_{j}\right\|_{\sim}^{2} \leq\left\|r_{j}\right\|_{2}^{2}$.

(c) If $\left|c_{i_{j}}\right|^{2}<\theta\left\|r_{j}\right\|_{\sim}^{2}$, break (out of for-loop)

(d) Output $t_{j}$

5. Encode the pseudorandom seeds for $R_{1}$ and $R_{2}$ into the low-order bits of the output or (as we assume here) provide $R_{1}$ and $R_{2}$ as auxiliary output.

Protocol 2. Protocol for the Euclidean Heavy Hitters problem

The difficulty arises when $\left|Q_{c, B, \theta}\right|<B$, in which case some of $I_{B}$ may be arbitrary and should not be allowed to leak. So the algorithm needs to find a private subset $I_{\text {out }}$ with $Q_{c, B, \theta} \subseteq I_{\text {out }} \subseteq I_{B}$. The challenge is subtle. Let $s$ denote $\left|Q_{c, B, \theta}\right|$. If the algorithm knew $s$, the algorithm could easily output $Q_{c, B, \theta}$, which is the indices of the top $s$ terms, a correct and private output. Unfortunately, determining $Q_{c, B, \theta}$ or $s=\left|Q_{c, B, \theta}\right|$ requires $\Omega(N)$ communication (see Section [5.2), so we cannot hope to find $Q_{c, B, \theta}$ exactly. Non-private norm estimation can be used to find a subset $I_{\text {out }}$ with $Q_{c, B, \theta} \subseteq I_{\text {out }} \subseteq Q_{c, B, \frac{\theta}{1+\epsilon}} \subseteq I_{\text {opt }}$, 
which is correct, but not quite private. Given $\left|I_{\text {out }}\right|$, the contents of $I_{\text {out }} \subseteq I_{\text {opt }}$ are indeed private, but the size of $I_{\text {out }}$ is, generally, non-private. Fortunately, if we use a private protocol for norm estimation, $\left|I_{\text {out }}\right|$ remains private. We now proceed to a formal analysis.

Theorem 17. Protocol PrIVAte_heavy_hitters requires $\operatorname{poly}(N, \log (M)$, $B, k, 1 / \epsilon)$ local computation, poly $(\log (N), \log (M), B, k, 1 / \epsilon)$ communication, and $O(1)$ rounds.

Proof. By existing work, all costs of Steps 1 to 3 are as claimed. Now consider Step 4. Observe that the function being computed there has inputs and outputs of size bounded by poly $(\log (N), \log (M), B, k, 1 / \epsilon)$ and takes time polynomial in the size of its inputs. In particular, the instances of NORM_ESTIMATION do not start from scratch with respect to $a$ or $b$; rather, they pick up from the precomputed short sketches $R_{2} a$ and $R_{2} b$. It follows that this function can be wrapped with SMC, preserving the computation and communication up to polynomial blowup in the size of the input and keeping the round complexity to $O(1)$.

We now turn to correctness and privacy. Let $I_{\text {out }}$ denote the set of indices corresponding to the set $T_{\text {out }}$ of output terms.

Theorem 18. Protocol PRIVATE_HEAVY_HITTERS is correct.

Proof. The correctness of Steps 2 and 3 follows from previous work. In Step 4 , we first show that $Q_{B, \frac{\epsilon}{B(1+\epsilon)}} \subseteq I_{\text {out }}$. We assume that $\frac{1}{1+\epsilon}\left\|r_{j}\right\|_{2}^{2} \leq\left\|r_{j}\right\|_{\sim}^{2} \leq\left\|r_{j}\right\|_{2}^{2}$ always holds; by Proposition [16] this happens with high probability. Thus, if $\left|c_{i_{j}}\right|^{2} \geq \frac{\epsilon}{B(1+\epsilon)}\left\|r_{j}\right\|_{2}^{2}$, then $\left|c_{i_{j}}\right|^{2} \geq \frac{\epsilon}{B(1+\epsilon)}\left\|r_{j}\right\|_{2}^{2} \geq \frac{\epsilon}{B(1+\epsilon)}\left\|r_{i}\right\|_{\sim}^{2}$. By construction, $Q_{B, \frac{\epsilon}{B(1+\epsilon)}} \subseteq I$. A straightforward induction shows that, if $j \in Q_{B, \frac{\epsilon}{B(1+\epsilon)}}$, then iteration $j$ outputs $t_{i_{j}}$ and the previous iterations output exactly the set of the $j$ larger terms in $I$. By Proposition 4, since $I_{\text {out }}$ is a superset of $Q_{B, \frac{\epsilon}{B(1+\epsilon)}}$, if $\widetilde{c}=\sum_{j \in I_{\mathrm{out}}} c_{i_{j}} \delta_{i_{j}}$, then $\|\widetilde{c}-c\|_{2}^{2} \leq(1+\epsilon)\left\|c_{\mathrm{opt}}-c\right\|_{2}^{2}$, as desired.

Before giving the complete privacy argument, we give a lemma, similar to the above. Suppose a set $P$ of indices is a subset of another set $Q$ of indices. We will say that $P$ is a prefix of $Q$ if $i \in P, t_{j}>t_{i}$, and $j \in Q$ imply $j \in P$.

Lemma 2. Output set $I_{\mathrm{out}}$ is a prefix of $Q_{B, \frac{\epsilon}{B(1+\epsilon)^{2}}}$ except with probability $2^{-k}$. Proof. Note that $Q_{B, \frac{\epsilon}{B(1+\epsilon)^{2}}}$ is a subset of $I$ and $Q_{B, \frac{\epsilon}{B(1+\epsilon)^{2}}}$ is a prefix of the universe, so $Q_{B, \frac{\epsilon}{B(1+\epsilon)^{2}}}$ is a prefix of $I$. The set $I_{\text {out }}$ is also a prefix of $I$. Thus, of the sets $I_{\text {out }}$ and $Q_{B, \frac{\epsilon}{B(1+\epsilon)^{2}}}$, one is a prefix of the other (or they are equal).

So suppose, toward a contradiction, that $Q_{B, \frac{\epsilon}{B(1+\epsilon)^{2}}}$ is a proper prefix of $I_{\text {out }}$. Let $q=\left|Q_{B, \frac{\epsilon}{B(1+\epsilon)^{2}}}\right|$, so $q$ is the least number such that $i_{q}$ is not in $Q_{B, \frac{\epsilon}{B(1+\epsilon)^{2}}}$. If the protocol halts before considering $q$, then $I_{\text {out }} \subseteq Q_{B, \frac{\epsilon}{B(1+\epsilon)^{2}}}$, a contradiction. So we may assume that $q<B$ (so the for-loop doesn't terminate). Then, by definition of $Q_{B, \frac{\epsilon}{B(1+\epsilon)^{2}}}$, we have $\left|c_{i_{q}}\right|^{2}<\frac{\epsilon}{B(1+\epsilon)^{2}} \sum_{j \geq q}\left|c_{i_{j}}\right|^{2}$. It follows that 
$\left|c_{i_{q}}\right|^{2}<\frac{\epsilon}{B(1+\epsilon)^{2}} \sum_{i \geq q}\left|c_{i}\right|^{2}=\frac{\epsilon}{B(1+\epsilon)^{2}}\left\|r_{q}\right\|_{2}^{2} \leq \frac{\epsilon}{B(1+\epsilon)}\left\|r_{q}\right\|_{\sim}^{2}$. Thus the protocol halts without outputting $t_{q}$, after outputting exactly $Q_{B, \frac{\epsilon}{B(1+\epsilon)^{2}}}$.

Finally, Theorem 19 ensures privacy and Theorem 20 summarizes our results.

Theorem 19. Protocol Private_HeAvy_hitTers leaks only $\|c\|_{2}^{2}$ and $c_{\mathrm{opt}}$.

Proof. With the random inputs $R_{1}$ and $R_{2}$ encoded into the output, it is straightforward to show that Protocol PRIVATE_HEAVY_HITTERS is a private protocol in the traditional sense that the protocol messages leak no more than the inputs and outputs. This is done by composing simulators for PRIVATE-SAMPLE-SUM and SMC. It remains only to show only that we can simulate the joint distribution on $\left(\widetilde{c}, R_{1}, R_{2}\right)$ given as simulator-input $c_{\mathrm{opt}}$ and $\|c\|$. We will show that $R_{1}$ is indistinguishable from independent of the joint distribution of $\left(\widetilde{c}, R_{2}\right)$, which we will simulate directly.

First, we show that $R_{1}$ is independent. Except with probability $2^{-\Omega(k)}$, the intermediate set $I$ is a superset of $Q_{B, \frac{\epsilon}{B(1+\epsilon)^{2}}}$ and the norm estimation is correct. In that case, the protocol outputs a prefix of $Q_{B, \frac{\epsilon}{B(1+\epsilon)^{2}}}$ and we get identical output if $I$ is replaced by $Q_{B, \frac{\epsilon}{B(1+\epsilon)^{2}}}$. Also, $Q_{B, \frac{\epsilon}{B(1+\epsilon)^{2}}}$ can be constructed from $c_{\mathrm{opt}}$ and $\|c\|_{2}$. Since the protocol proceeds without further reference to $R_{1}$, we have shown that the pair $\left(\widetilde{c}, R_{2}\right)$ is indistinguishable from being independent of $R_{1}$. It remains only to simulate $\left(\widetilde{c}, R_{2}\right)$.

Note that the output $\widetilde{c}$ does depend non-negligibly on $R_{2}$. If $\left|c_{i_{j}}\right|^{2}$ is very close to $\theta\left\|r_{j}\right\|_{2}^{2}$, then the test $\left|c_{i_{j}}\right|^{2}<\theta\left\|r_{j}\right\|_{\sim}^{2}$ in the protocol may succeed with probability non-negligibly far from 0 and from 1 , depending on $R_{2}$, since the distortion guarantee on $\left\|r_{j}\right\|_{\sim}^{2}$ is only the factor $(1 \pm \epsilon)$.

The simulator is as follows. Assume that the terms in $c_{\text {opt }}$ are $t_{0}, t_{1}, \ldots, t_{B-1}$ with decreasing order, $t_{0}>t_{1}>\cdots>t_{B-1}$. For each $j \leq B$, compute $E_{j}=\left\|c-\left(t_{0}+t_{1}+\cdots+t_{j-1}\right)\right\|_{2}^{2}=\|c\|_{2}^{2}-\left\|t_{0}+t_{1}+\cdots+t_{j-1}\right\|_{2}^{2}$ and then run the NORM_ESTIMATION simulator on input $E_{j}$ and $\epsilon$ to get a sample from the joint distribution $\left(\widetilde{E}_{j}, \bar{R}_{2}\right)$, where $\widetilde{E}_{j}$ is a good estimate to $E_{j}$. Our simulator then outputs $t_{i_{j}}$ if $\left|c_{i_{j}}\right|^{2} \geq \frac{\epsilon}{B(1+\epsilon)} \widetilde{E}_{j}$, and halts, otherwise, following the final for-loop of the protocol. Call the output of the simulator $\widetilde{s}=\sum_{j} t_{i_{j}} \delta_{i_{j}}$.

Again using the fact that a prefix of $Q_{B, \frac{\epsilon}{B(1+\epsilon)^{2}}}$ is output, if $j \in Q_{B, \frac{\epsilon}{B(1+\epsilon)^{2}}}$, then $i_{j}=j$; i.e., the $j^{\text {th }}$ largest output term is the $j^{\text {th }}$ largest overall, so that, if $j$ is output, $E_{j}=\left\|r_{j}\right\|_{2}^{2}$. Thus $\left(\widetilde{E}_{j}, \bar{R}_{2}\right)$ is distributed indistinguishably from $\left(\left\|r_{j}\right\|_{\sim}^{2}, R_{2}\right)$. The protocol finishes deterministically using $I$ and $\left\|r_{j}\right\|_{\sim}^{2}$ and the simulator finishes deterministically using $Q_{B, \frac{\epsilon}{B(1+\epsilon)^{2}}}$ and $\widetilde{E}_{j}$, but, since the protocol output is identical if $I$ is replaced by $Q_{B, \frac{\epsilon}{B(1+\epsilon)^{2}}}$, the distributions on output $\left(\widetilde{c}, R_{2}\right)$ of the protocol and $\left(\widetilde{s}, \bar{R}_{2}\right)$ of the simulator are indistinguishable.

Theorem 20. Suppose Alice and Bob hold integer-valued vectors $a$ and $b$ in $[-M, M]^{N}$, respectively. Let $B, k$ and $\epsilon$ be user-defined parameters. Let $c=$ $a+b$. Let $T_{\mathrm{opt}}$ be the set of the largest $B$ terms in $c$. There is a protocol, taking $a, b, B k$ and $\epsilon$ as input, that computes a representation $\widetilde{c}$ of at most $B$ 
terms such that it: (a) outputs $\widetilde{c}$ with $\|\widetilde{c}-c\|_{2} \leq(1+\epsilon)\left\|c_{\mathrm{opt}}-c\right\|_{2}$; (b) uses $\operatorname{poly}(N, \log (M), B, k, 1 / \epsilon)$ time, $\operatorname{poly}(\log (N), \log (M), B, k, 1 / \epsilon)$ communication, and $O(1)$ rounds; and (c) succeeds with probability $1-2^{-k}$ and leaks only $c_{\mathrm{opt}}$ and $\|c\|_{2}$ on security parameter $k$.

Corollary 21. With the same hyptotheses and resource bounds, there is a protocol that computes $\widetilde{c}$ and an approximation $\|\widetilde{c}-c\|_{\sim}$ to $\|\widetilde{c}-c\|_{2}$ such that $\frac{1}{1+\epsilon}\|\widetilde{c}-c\|_{2} \leq\|\widetilde{c}-c\|_{\sim} \leq\|\widetilde{c}-c\|_{2}$ and the protocol leaks only $c_{\mathrm{opt}}$ and $\|\widetilde{c}-c\|_{2}$.

Proof. Run the main protocol and output also $\|\widetilde{c}-c\|_{\sim}$, computed in the course of the main protocol. Note that $\|\widetilde{c}-c\|_{2}^{2}=\|c\|_{2}^{2}-\|\widetilde{c}\|_{2}^{2}$ and both $\|c\|_{2}$ and $\widetilde{c}$ are available to the main simulator (as input and output, resp.), so we can modify the main simulator to compute $\|\widetilde{c}-c\|_{2}^{2}$ as well.

\subsection{Lower Bounds}

In this Section, we state some lower bounds for problems related to our main problem in this Section, such as computing an approximation to $c_{\text {opt }}$ without leaking $\|c\|_{2}$. The results are straightforward, but we include Theorem 22 to motivate the approximation and Theorem 23 to motivate leakage of the Euclidean norm in protocols we present. The proofs, based on the set disjointness problem, will appear in the journal version of this article.

Theorem 22. There is an infinite family of settings of parameters $M, N, B, k$ such that any protocol that computes the Euclidean norm exactly on the sum $c$ of individually-held inputs a and $b$, uses communication $\Omega(N)$. Similarly, any protocol that computes the exact Heavy Hitters or computes the qualified set $Q_{c, 1,1}$ exactly uses communication $\Omega(N)$.

Theorem 23. There is an infinite family of settings of parameters $M, N, B, k, \epsilon$ such that any protocol that solves the Euclidean Heavy Hitters problem on the sum $c$ of individually-held inputs $a$ and $b$, leaking only $c_{\mathrm{opt}}$, uses communication $\Omega(N)$. Furthermore, for an infinite class of inputs in which $\|c\|_{2}$ is not constant, any such protocol either computes $\|c\|_{2}$ or uses communication $\Omega(N)$.

\section{References}

1. Ailon, N., Chazelle, B.: Approximate nearest neighbors and the fast JohnsonLindenstrauss transform. In: Proc. 38th Annual ACM STOC, pp. 557-563 (2006)

2. Aggarwal, G., Mishra, N., Pinkas, B.: Secure computation of the k th-ranked element. In: Cachin, C., Camenisch, J.L. (eds.) EUROCRYPT 2004. LNCS, vol. 3027, pp. 40-55. Springer, Heidelberg (2004)

3. Alon, N., Gibbons, P.B., Matias, Y., Szegedy, M.: Tracking join and self-join sizes in limited storage. J. Comput. Syst. Sci. 64(3), 719-747 (2002)

4. Alon, N., Matias, Y., Szegedy, M.: The space complexity of approximating the frequency moments. J. Comput. Syst. Sci. 58(1), 137-147 (1999) 
5. Beimel, A., Carmi, P., Nissim, K., Weinreb, E.: Private approximation of search problems. In: Proc. 38th Annual ACM STOC, pp. 119-128 (2006)

6. Ben-Or, M., Goldwasser, S., Wigderson, A.: Completeness theorems for noncryptographic fault-tolerant distributed computation. In: Proc. 20th Annual ACM STOC, pp. 1-10. ACM Press, New York (1988)

7. Cachin, C., Micali, S., Stadler, M.: Computationally private information retrieval with polylogarithmic communication. In: Stern, J. (ed.) EUROCRYPT 1999. LNCS, vol. 1592, pp. 404-414. Springer, Heidelberg (1999)

8. Chawla, S., Dwork, C., McSherry, F., Smith, A., Wee, H.: Toward privacy in public databases. In: Kilian, J. (ed.) TCC 2005. LNCS, vol. 3378, pp. 363-385. Springer, Heidelberg (2005)

9. Chor, B., Goldreich, O., Kushilevitz, E., Sudan, M.: Private information retrieval. Journal of the ACM 45, 965-981 (1998)

10. Cormode, G., Muthukrishnan, S.: What's hot and what's not: Tracking most frequent items dynamically. In: Proc. ACM PODS, pp. 296-306 (2003)

11. Feigenbaum, J., Ishai, Y., Malkin, T., Nissim, K., Strauss, M., Wright, R.N.: Secure multiparty computation of approximations. Transactions on Algorithms (2006). An extended abstract appeared in ICALP 2001

12. Freedman, M., Nissim, K., Pinkas, B.: Efficient private matching and set intersection. In: Cachin, C., Camenisch, J.L. (eds.) EUROCRYPT 2004. LNCS, vol. 3027, pp. 1-19. Springer, Heidelberg (2004)

13. Gilbert, A., Guha, S., Indyk, P., Kotidis, Y., Muthukrishnan, S., Strauss, M.: Fast, small-space algorithms for approximate histogram maintenance. In: Proc. 34th Annual ACM STOC, pp. 389-398 (2002)

14. Halevi, S., Kushilevitz, E., Krauthgamer, R., Nissim, K.: Private approximations of NP-hard functions. In: Proc. 33th Annual ACM STOC, pp. 550-559 (2001)

15. Indyk, P., Woodruff, D.P.: Polylogarithmic private approximations and efficient matching. In: Proc. Third Theory of Cryptography Conference, pp. 245-264 (2006)

16. Kushilevitz, E., Mansour, Y.: Learning decision trees using the fourier sprectrum. In: Proc. 23th Annual ACM STOC, pp. 455-464 (1991)

17. Kushilevitz, E., Nisan, N.: Communication complexity. Cambridge University Press, Cambridge (1997)

18. Kushilevitz, E., Ostrovsky, R.: Replication is NOT needed: SINGLE database, computationally-private information retrieval. In: Proc. 38th IEEE FOCS, pp. 364373 (1997)

19. Lindell, Y., Pinkas, B.: Privacy preserving data mining. J. Cryptology 15(3), $177-$ $206(2002)$

20. Milman, V.D., Schechtman, G.: Asymptotic Theory of Finite Dimensional Normed Spaces. Lecture Notes in Mathematics, vol. 1200 (1986)

21. Naor, M., Nissim, K.: Communication preserving protocols for secure function evaluation. In: Proc. 33th Annual ACM STOC, pp. 590-599 (2001)

22. Yao, A.: Protocols for secure computation. In: Proc. 23rd IEEE FOCS, pp. 160-164 (1982) 